\title{
Brain Oscillations Control Timing of Single-Neuron Activity in Humans
}

\author{
Joshua Jacobs, ${ }^{1}$ Michael J. Kahana, ${ }^{2}$ Arne D. Ekstrom, ${ }^{3}$ and Itzhak Fried ${ }^{4,5}$ \\ ${ }^{1}$ Neuroscience Graduate Group and ${ }^{2}$ Department of Psychology, University of Pennsylvania, Philadelphia, Pennsylvania 19104, ${ }^{3}$ Center for Cognitive \\ Neurosciences, Semel Institute, Department of Psychiatry and Biobehavioral Sciences, University of California School of Medicine, Los Angeles, California \\ 90095, ${ }^{4}$ Division of Neurosurgery and Semel Institute for Neuroscience and Human Behavior, University of California, Los Angeles, California 90095, and \\ ${ }^{5}$ Functional Neurosurgery Unit, Tel-Aviv Medical Center and Sackler Faculty of Medicine, Tel-Aviv University, Tel-Aviv 69978, Israel
}

A growing body of animal research suggests that neurons represent information not only in terms of their firing rates but also by varying the timing of spikes relative to neuronal oscillations. Although researchers have argued that this temporal coding is critical in human memory and perception, no supporting data from humans have been reported. This study provides the first analysis of the temporal relationship between brain oscillations and single-neuron activity in humans. Recording from 1924 neurons, we find that neuronal activity in various brain regions increases at specific phases of brain oscillations. Neurons in widespread brain regions were phase locked to oscillations in the theta- $(4-8 \mathrm{~Hz})$ and gamma- $(30-90 \mathrm{~Hz})$ frequency bands. In hippocampus, phase locking was prevalent in the delta$(1-4 \mathrm{~Hz})$ and gamma-frequency bands. Individual neurons were phase locked to various phases of theta and delta oscillations, but they only were active at the trough of gamma oscillations. These findings provide support for the temporal-coding hypothesis in humans. Specifically, they indicate that theta and delta oscillations facilitate phase coding and that gamma oscillations help to decode combinations of simultaneously active neurons.

Key words: phase locking; theta; gamma; intracranial EEG; navigation; local field potential

\section{Introduction}

Many recent neurobiological theories of human memory and perception rely critically on brain oscillations to coordinate the timing of neuronal spiking. For example, Buzsáki (2005) proposed a theory of episodic memory in which theta oscillations $(4-8 \mathrm{~Hz})$ act as a timing signal to ensure that neurons representing related stimuli spike nearby each other in time. In addition, other researchers have suggested memory models that rely on theta for different functions such as separating the memoryencoding and the memory-retrieval processes (Hasselmo et al., 2002) or maintaining stimuli representations in working memory (Lisman and Idiart, 1995). In addition to theta, gamma oscillations $(30-90 \mathrm{~Hz})$ play a role in a different set of models in which they bind together sets of neurons to represent complex stimuli (for review, see Fries, 2005).

The experimental data supporting these theories come from simultaneous recordings of neuronal spiking and local-field potentials (LFPs) in animals. For example, the discovery that some rodent hippocampal neurons spike at different phases of the theta

\footnotetext{
Received 0ct. 25, 2006; revised Jan. 26, 2007; accepted Feb. 20, 2007.

This work was supported by National Institutes of Health Research Grants MH61975 and MH062196, National Science Foundation Grant SBE0354378, and by the Swartz Foundation. We gratefully acknowledge help from Matthew Mollison and Igor Korolev for cluster cutting and Sean Polyn, Marieke van Vugt, and Per Sederberg for helpfu discussions.

Correspondence should be addressed to either of the following: Dr. Itzhak Fried, Division of Neurosurgery, University of California, Los Angeles, Los Angeles, CA 90095, E-mail: ifried@mednet.ucla.edu; or Dr. Michael J. Kahana, Department of Psychology, University of Pennsylvania Philadelphia, PA 19104, E-mail: kahana@psych.upenn.edu. D0I:10.1523/JNEUROSCI.4636-06.2007

Copyright $\odot 2007$ Society for Neuroscience $\quad$ 0270-6474/07/273839-06\$15.00/0
}

oscillation depending on the animal's behavioral state or spatial location suggested that theta may be a general mechanism for phase coding (Fox et al., 1986; O'Keefe and Recce, 1993). There is also considerable evidence for the role of gamma oscillations in animal cognition and behavior. For example, in olfactory and visual cortices, the amplitude and synchrony of gamma oscillations indicates properties of external stimuli (Freeman and Schneider, 1982; Gray et al., 1989). Furthermore, recent research indicates that gamma oscillations play a role in cognitive behaviors such as maintaining stimuli representations in working memory (Pesaran et al., 2002) and selectively attending to one visual stimulus in a crowded scene (Fries et al., 2001). Although excitatory and inhibitory neurons spike at different phases of the gamma oscillation (Eeckman and Freeman, 1990; Csicsvari et al., 2003), there are no reports of gamma-band phase coding in which a single neuron would spike at different phases of gamma according to behavior.

Consistent with these striking observations of brain oscillations in animals, there is now a large body of work demonstrating functional correlates of theta and gamma oscillations in the human brain (for review, see Kahana, 2006). These studies have used a variety of methods including scalp electroencephalography and magnetoencephalography, as well as LFPs recorded intracranially in patients undergoing neurosurgical treatments. Although brain oscillations, particularly in the theta band, are prominent both in humans and in animals, it has sometimes proven difficult to link human and animal research findings. The theta oscillation most often studied in humans is in the neocortex 
and has many distinct local generators (Raghavachari et al., 2006), whereas the theta oscillation typically studied in animals is in the hippocampus and is primarily driven by one of a small number of sources (Buzsáki, 2002). To date, no human studies have compared brain oscillations to the timing of neuronal spiking.

Our goal in the present study was to characterize the temporal relationship between single-neuron activity and LFP oscillations in the human brain. Going beyond previous animal work, we sought to characterize this relationship across a wide range of frequencies and across a number of disparate brain regions. To accomplish this, we recorded simultaneous spiking activity and LFPs from intracranial microelectrodes in 20 patients undergoing treatment for drug-resistant epilepsy (see Materials and Methods). In these recordings, we identified a total of 1924 neurons, and compared the activity of each neuron with LFP oscillations recorded at the same microelectrode. During recording, subjects played "Yellow Cab," a virtual taxi driver video game. We selected this task because it induces task-related brain oscillations at a range of frequencies, including the theta band, in cortical and limbic structures (Ekstrom et al., 2005).

\section{Materials and Methods}

We examined data from intracranial microelectrodes in patients undergoing surgical treatment for drug-resistant epilepsy. Electrodes were positioned by clinical teams to isolate the epileptic seizure focus for subsequent potential surgical resection (surgeries performed by I.F.). Microelectrode coverage included hippocampus (377 neurons), parahippocampal region (Witter and Wouterlood, 2002) (421 neurons), amygdala (404 neurons), frontal regions (438 neurons), and occasionally temporal and parietal cortices (284 neurons). This study conformed to the guidelines of the Medical Institutional Review Board at University of California, Los Angeles. We examined data from a total of 46 recording sessions from 20 different subjects (individual subjects participated in 1-4 sessions) (for details, see supplemental Table 1, available at www. jneurosci.org as supplemental material). These recording sessions took place in patients' free time between medical treatments. During each recording session, subjects played "Yellow Cab" (Ekstrom et al., 2005) on a bedside laptop computer for $25-60 \mathrm{~min}$. During the game, $\approx 75 \%$ of the participants' time was spent actively moving to particular landmarks in the environment. The remainder of the time was spent reading instruction screens and planning routes.

Each patient was implanted with 6-12 clinical intracranial depth electrodes. Each clinical electrode terminated with a set of nine $40 \mu \mathrm{m}$ platinum-iridium microwires (Fried et al., 1999). The first eight microwires were insulated except for their tip and were used to record single-unit action potentials and LFPs. The ninth microwire had its insulation stripped for $\approx 1 \mathrm{~cm}$ and served as the recording reference for the other eight microwires on that depth probe. We recorded from each microwire at sampling rates of $28-32 \mathrm{kHz}$ using a Cheetah recording system (Neuralynx, Tucson, AZ). Action potentials were manually isolated using spike shape, clustering of wavelet coefficients, and interspike intervals (Quiroga et al., 2004). Typically we isolated zero or one distinct neuron from each microwire, but in rare cases we observed up to three distinct neurons from a single microwire.

For analyses of LFP oscillations, we downsampled recordings to $2 \mathrm{kHz}$ and then applied 60 and $120 \mathrm{~Hz}$ second-order Butterworth notch filters. To minimize the contribution of low-frequency components of spikes toward spectral calculations, we removed the samples from $2 \mathrm{~ms}$ before to $8 \mathrm{~ms}$ after each spike and replaced them with a linear interpolation of the underlying LFP signal. Then, we computed oscillatory phase and power of the LFP using Morlet wavelets (wave number, 4) at frequencies between 1 and $100 \mathrm{~Hz}\left(2^{x / 8} \mathrm{~Hz}\right.$ for $\left.x \in 0, \ldots, 53\right)$. We considered a neuron phase locked at frequency $f$ if the hypothesis of uniformity for its LFP $f$ hertz phase distribution could be rejected at $p<0.001$ using a Bonferroni-corrected Rayleigh test (Fisher, 1993). This Bonferroni correction compensated for our application of the Rayleigh test across each of 54 frequencies. In summary statistics, if a neuron fulfilled this phaselocking criterion at multiple frequencies, we considered it phase locked at the frequency at which its phase distribution was most nonuniform according to the Rayleigh statistic.

We studied neuronal spiking activity during different levels of LFP oscillatory power by first calculating the phase and power of the LFP of each phase-locked neuron throughout the entire recording session, at the frequency at which it was phase locked. We then labeled each point of the recording session according to whether the LFP exhibited high, medium, or low power. To perform this labeling, first we removed any potential artifacts caused by movement or electrical noise by discarding the $10 \%$ of the recording session corresponding to the lowest fifth percentile and the highest 95th percentile of LFP power throughout the recording session. Then, we labeled the remaining time points into one of three groups (high, medium, or low) according to the instantaneous log-transformed power (at the phase-locked frequency). The boundaries for these groups were equally spaced across log-transformed power values. Finally, separately for the time points in each group, we calculated the instantaneous firing rate of each neuron as a function of oscillatory phase. (The phase was divided into 16 groups equally spaced between 0 and $2 \pi$.)

\section{Results}

We identified 1215 neurons whose spiking was phase locked to LFP oscillations. This phenomenon was prominent in the theta band $(4-8 \mathrm{~Hz})$. Figure $1 A$ illustrates the behavior of a neuron in the right superior temporal gyrus of subject 2 that was phase locked to $7.3 \mathrm{~Hz}$ theta oscillations. This firing rate of the neuron varied by more than twofold according to the instantaneous theta phase: it had a firing rate of $7.9 \mathrm{~Hz}$ at its preferred phase (5.7 rad) just before the peak of the oscillation and a firing rate of $3.7 \mathrm{~Hz}$ near the trough of the oscillation (Fig. $1 A$, right). Figure $1 B$ shows the activity of a neuron from subject 1's right temporal cortex that was phase locked to the trough of $6.2 \mathrm{~Hz}$ theta oscillations.

In addition to the theta band, we also identified neurons that exhibited phase-locked spiking to oscillations in the delta- (1-4 $\mathrm{Hz})$, alpha- $(10-16 \mathrm{~Hz})$, beta- $(16-30 \mathrm{~Hz})$, and gamma- $(30-90$ $\mathrm{Hz}$ ) frequency ranges. Thus, human neuronal phase locking is a general phenomenon that is not isolated to a narrow frequency range. For example, Figure 1, $C$ and $D$, depicts the activities of neurons in entorhinal and orbitofrontal cortices that were phase locked to oscillations at 3.4 and $3.7 \mathrm{~Hz}$, respectively. Figure 1, E and $F$, shows the behavior of hippocampal neurons that were phase locked to gamma oscillations.

An additional pattern we observed was that neurons could be phase locked to oscillations at multiple frequencies. For example, Figure $1 E$ shows the activity of a neuron from the right posterior hippocampus of subject 4 that was primarily phase locked to 70 Hz gamma oscillations but also exhibited moderate phase locking to delta oscillations. Figure $1 \mathrm{H}$ presents the activity of a neuron from the left amygdala of subject 3 that exhibited significant phase locking to both 9.5 and $1.8 \mathrm{~Hz}$ oscillations. Figure $1 C$ describes a neuron from the left entorhinal cortex that was phase locked to oscillations at 3.4 and $12 \mathrm{~Hz}$.

In the rodent hippocampus, the prominent oscillatory pattern is the $4-8 \mathrm{~Hz}$ theta rhythm (Buzsáki, 2002). However, in our recordings, we found that hippocampal phase locking was more prevalent in the $1-4 \mathrm{~Hz}$ range than in the $4-8 \mathrm{~Hz}$ range. For example, Figure $1 G$ depicts the activity of a hippocampal neuron that was phase locked to oscillations at $1.1 \mathrm{~Hz}$. In addition, the hippocampal neurons highlighted in Figure 1, $E$ and $F$, also exhibit significant phase locking at $1-4 \mathrm{~Hz}$.

Animal studies have identified neurons that were phase locked to local oscillations in hippocampus (Fox et al., 1986; Skaggs et al., 1996; Csicsvari et al., 2003) and entorhinal (Chrobak and 

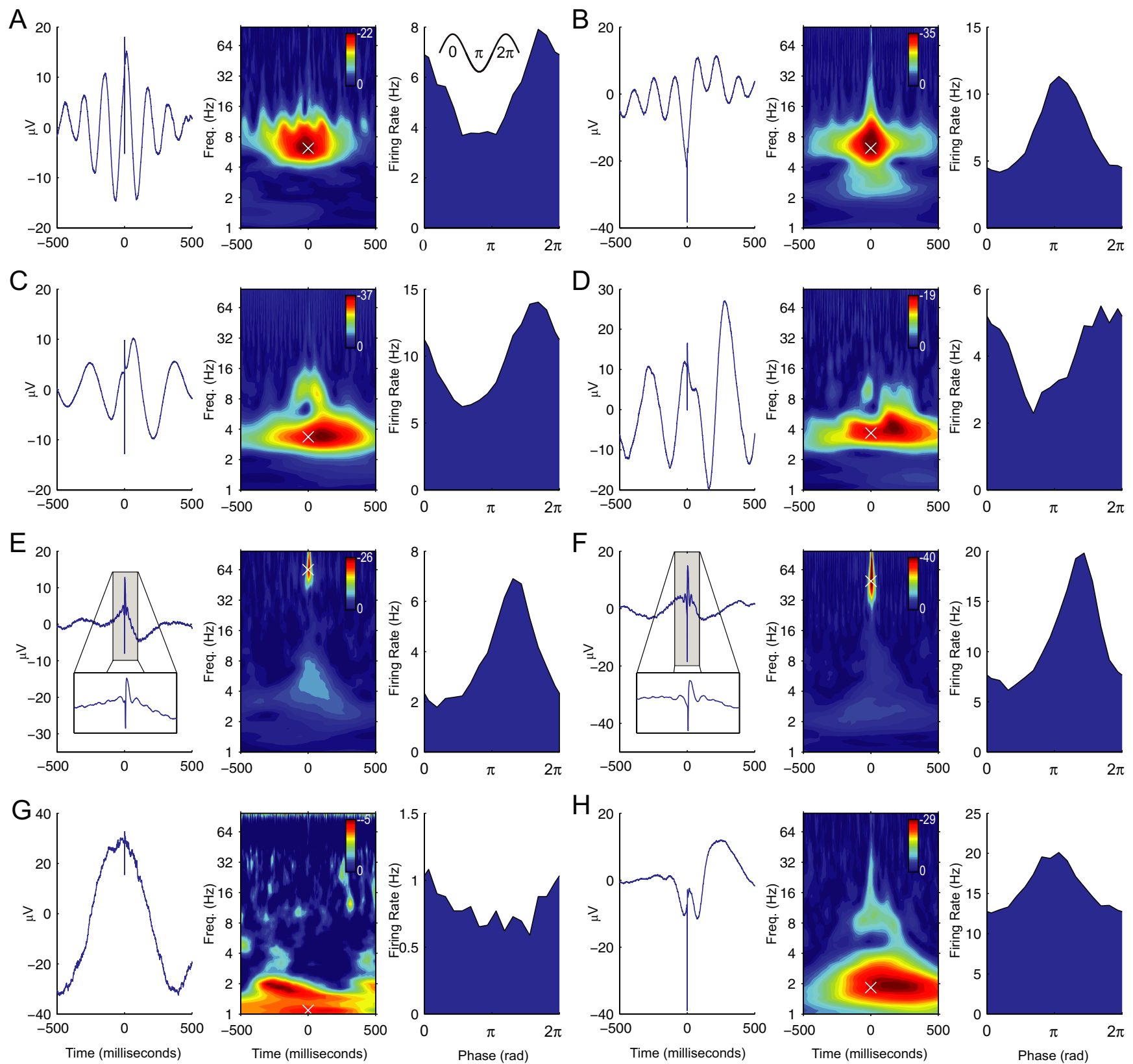

Figure 1. A, Activity of a neuron from subject 2's right superior temporal gyrus, which was phase locked to the peak of $7.3 \mathrm{~Hz}$ theta oscillations. Left, Spike-triggered LFP average. Spike onset occurred at $0 \mathrm{~ms}$. Middle, Zscore from Rayleigh test evaluating the hypothesis of a uniform LFP phase distribution at the moment of spike onset. Shifts along the horizontal axis indicate the relation between spiking and phase of time-shifted LFP oscillations. The white $\times$ symbol indicates the frequency at which phase locking at 0 ms is most statistically significant. Right, Firing rate as a function of phase at $7.3 \mathrm{~Hz}$ (the frequency marked by the $X$ in middle panel). The circular mean resultant vector length $\bar{R}$ (Fisher, 1993) of this $7.3 \mathrm{~Hz}$ LFP phase distribution is 0.19 . The inset example waveform is a reminder that the phases of the peak and trough of an oscillation are 0 (or $2 \pi$ ) radians and $\pi$ radians, respectively. $B$, Activity of a neuron from subject 1 's right temporal gyrus that was phase locked to the trough of $6.2 \mathrm{~Hz}$ theta oscillations $(\bar{R}=0.24)$. C, Behavior of a neuron from subject 20's left entorhinal cortex, which was primarily phase locked to $3.4 \mathrm{~Hz}$ oscillations $(\bar{R}=$ $0.2)$, but also exhibited some phase locking to oscillations at $9.5 \mathrm{~Hz}$. D, A neuron from subject 18 's right orbitofrontal cortex, which was phase locked to $3.7 \mathrm{~Hz}$ oscillations $(\bar{R}=0.17) . E$, Behavior of a neuron from subject 4's left posterior hippocampus that was phase locked to the trough of $70 \mathrm{~Hz}$ gamma oscillations $(\bar{R}=0.17)$. Inset in left panel depicts a zoomed plot of the spike-triggered average of this neuron. $\boldsymbol{F}$, Behavior of a neuron from subject 12 's left anterior hippocampus that exhibited phase-locked spiking near the trough of $49 \mathrm{~Hz}$ gamma oscillations $(\bar{R}=0.2) . \mathbf{G}$, The activity of a neuron from subject 1's left anterior hippocampus, which was phase locked to the peak of $1.1 \mathrm{~Hz}$ oscillations $(\bar{R}=0.1)$. $\boldsymbol{H}$, Activity of a neuron from the left amygdala of subject 3 that was phase locked to oscillations primarily at $1.8 \mathrm{~Hz}(\bar{R}=0.11)$, but also at $9.5 \mathrm{~Hz}$.

Buzsáki, 1998), parietal (Pesaran et al., 2002), and visual cortices (Fries et al., 2001; Lee et al., 2005): this phenomenon was notably absent from frontal cortex (Siapas et al., 2005). Because our dataset included recordings from widespread brain regions, we had the opportunity to determine whether the prevalence of phase locking in particular frequency ranges localized to particular brain regions. We found that the prevalence of phase locking significantly varied across brain regions in the delta- $[\chi 2(4)=89$; $p<0.001]$, theta- $[\chi 2(4)=78 ; p<0.001]$, and gamma- $[\chi 2(4)=$ $71 ; p<0.001]$ frequency bands. (For details, see supplemental Table 1, available at www.jneurosci.org as supplemental material.) Figure $2 \mathrm{~A}$ shows that phase locking was especially prevalent at theta frequencies in temporal and parietal cortices $[\chi 2(1)=64$, $p<0.001$, Bonferroni-corrected] and at delta frequencies in hippocampus $[\chi 2(1)=47, p<0.001$, Bonferroni-corrected $]$. In hippocampus, significantly more neurons were phase locked to 
delta oscillations than to theta oscillations $[\chi 2(1)=51 ; p<0.001]$. Note that unlike some recent studies in animals (Siapas et al., 2005), we report some phase locking to theta oscillations in orbitofrontal cortices (20 of 438 cells) (see also Fig. 1D). Figure $2 C$ depicts the strength of phase locking across all phase-locked neurons by plotting the circular mean resultant vector length $\bar{R}$, which is a measure of relative increase in firing rate of each neuron at its preferred phase (Fisher, 1993). The median $\bar{R}$ across the population of phaselocked neurons is 0.1 .

The phase-coding hypothesis predicts that neurons encode information via the oscillatory phase at which they spike (Huxter et al., 2003). This theory thus predicts that different neurons would spike at varied phases of ongoing oscillations. To test the phase-coding hypothesis in humans, we labeled the preferred phase of each neuron as the oscillatory phase at which its firing rate was highest. (The peak and trough of each oscillation correspond to 0 [or $2 \pi$ ] radians and $\pi$ radians, respectively.) Figure $2 B$ shows the preferred phase of each phase-locked neuron. Neurons that were phase locked to delta or theta oscillations had varied preferred phases (Fig. 2D; see also Fig. $1 A-D, G, H$ ). However, neurons phase locked to gamma oscillations had preferred phases at or just after the trough of the oscillation (Fig. $2 \mathrm{E}$; see also Fig. $1 E, F)$. These dissimilar preferred-phase patterns $(p<0.001$,

Mardia-Watson-Wheeler test) indicate that neurons phase locked to gamma oscillations behave differently from those phase locked to delta or theta oscillations.

Although recent work indicates that the power of brain oscillations related to mean neuronal firing rates (Logothetis et al., 2001; Mukamel et al., 2005; Niessing et al., 2005), it is unknown how this phenomenon relates to the activity of phase-locked neurons. To study this relationship, we analyzed the firing rate of each phase-locked neuron during high-, medium-, and lowpower oscillations (see Materials and Methods). We identified a number of neurons that exhibited excitation (i.e., increased firing) at their preferred phase during high-power oscillations, compared with their activity during low oscillatory power (Fig. $3 A, B)$. Some neurons displayed the opposite pattern and were primarily inhibited (i.e., decreased firing) at nonpreferred phases during high-power oscillations (Fig. 3C). Finally, other neurons responded to high-power oscillations with substantial firing-rate changes at all phases (Fig. 3D).

This led us to ask whether, across all neurons, phase locking was primarily related to excitation at preferred phases or inhibition at nonpreferred phases. Our analysis showed that neurons exhibited significantly greater firing rates during high-power oscillations than during periods of low oscillatory power (mean increase, $0.7 \mathrm{~Hz} ; p<0.001$, $\mathrm{t}$ test). This effect was especially robust at the preferred phase of high-power oscillations at which the mean firing rate was elevated by $3.8 \mathrm{~Hz}$ compared with periods of low oscillatory power $(p<0.001, t$ test). This indicates
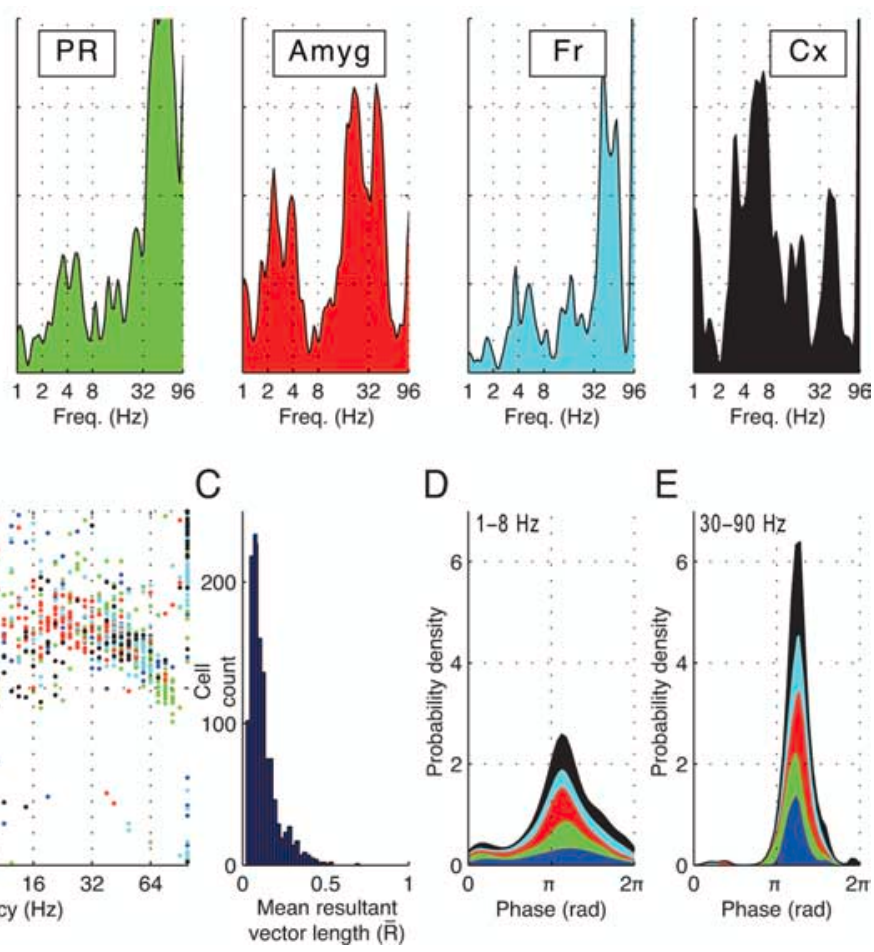

E

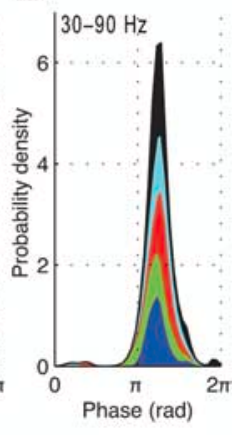

Figure 2. A, Probability of a neuron exhibiting significant phase locking as a function of frequency (Freq.), grouped by region. Materials and Methods for phase-locking criteria. Total area under each curve indicates the fraction of neurons in each region that probability density for all neurons that were phase locked at delta or theta frequencies $(1-8 \mathrm{~Hz})$. Coloring within each curve indicates the preferred-phase distributions of neurons in different regions. $\boldsymbol{E}$, Preferred-phase probability density for all neurons that were phase locked to gamma oscillations $(30-90 \mathrm{~Hz})$.

that the phase-locking phenomenon is primarily related to increased firing during high-power oscillations. This is consistent with the in vitro finding that some neurons act as bandpass filters by spiking in response to experimentally induced electrical oscillations at particular frequencies (Pike et al., 2000).

\section{Discussion}

Our results indicate that the neuronal phase-locking phenomenon is present in various brain regions of humans engaged in cognitive tasks. This phenomenon, in conjunction with the well, studied and diverse relationship between brain oscillations and human and animal learning (Berry and Thompson, 1978; Rizzuto et al., 2003; Jacobs et al., 2006), indicates that oscillationmodulated temporal coding plays a role in human cognition. Furthermore, our data suggest that gamma oscillations facilitate a different type of information coding compared with delta or theta oscillations. Delta and theta oscillations may facilitate phase-based temporal coding because we found that individual phase-locked neurons had widely varied preferred phases. This is consistent with the observations that different rodent hippocampal neurons were phase locked to varied theta phases (Fox et al., 1986; O'Keefe and Recce, 1993) and that the amplitude of gamma oscillations varies according to the instantaneous theta phase (Mormann et al., 2005). In contrast to delta and theta oscillations, neurons that phase locked to gamma oscillations consistently had preferred phases near the trough of the oscillation; thus, it seems unlikely that these oscillations facilitate phase cod- 
A

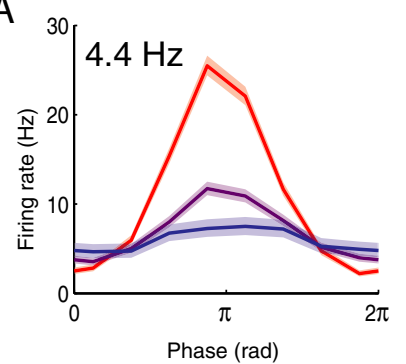

C

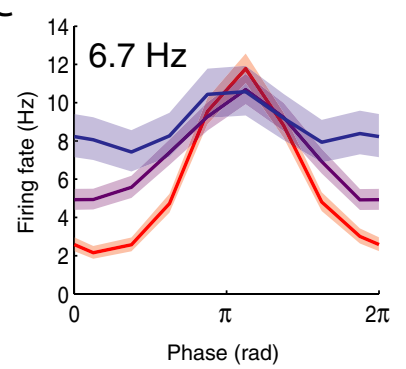

B

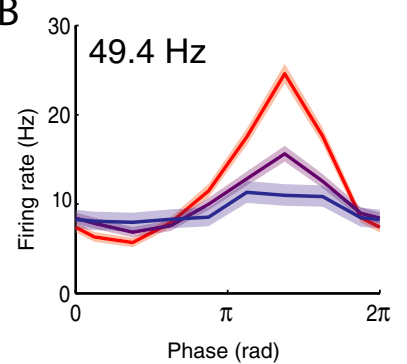

$\mathrm{D}$

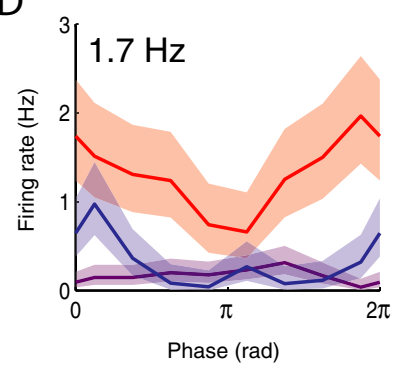

Figure 3. Relationship between oscillatory power and firing rates of phase-locked neurons. $A$, Activity of a neuron from subject 5 's left parietal cortex that exhibited maximal phase locking at $4.4 \mathrm{~Hz}$. Red, purple, and blue lines indicate firing rates during high, medium, and low oscillatory power, respectively (for details, see Materials and Methods). The shaded area indicates $95 \%$ confidence intervals based on the binomial distribution. $\boldsymbol{B}$, Activity of a neuron from subject 12 's left anterior hippocampus (same neuron as Fig. $1 F$ ) that was phase locked to LFP oscillations at $49 \mathrm{~Hz}$. C, Activity of a neuron from subject 1's right superior temporal gyrus (same neuron as Fig. 1 A). D, Activity of a neuron from subject 18 's left anterior hippocampus.

ing. This trough-locked spiking is similar to data from animal studies demonstrating that neurons in both neocortex and hippocampus spike at or just after the trough of gamma oscillations (Eeckman and Freeman, 1990; Csicsvari et al., 2003). In these systems, it is believed that information is encoded by the combination of neurons that spike at least once in each oscillation cycle, rather than the phase of each spike. Thus, our findings are consistent with the idea that human neuronal networks oscillating at gamma frequencies encode information by the combination of active neurons in each 10-50 ms oscillation cycle (Harris et al., 2003; Fries, 2005).

One important difference between our human results and animal electrophysiological literature is our observation of prominent hippocampal oscillations at $1-4 \mathrm{~Hz}$ (see Figs. $1 E-G, 2 A$ ) rather than the $4-8 \mathrm{~Hz}$ theta oscillation prominent in rodent hippocampus (Buzsáki, 2002). Although this human hippocampal oscillation falls into the delta range $(1-4 \mathrm{~Hz})$, it shares several characteristics with the $4-8 \mathrm{~Hz}$ rodent theta oscillation. For example, we found that individual hippocampal neurons were phase locked to widespread phases of this $1-4 \mathrm{~Hz}$ oscillation (Fig. $2 D$ ) and that some hippocampal neurons simultaneously phase locked both to these $1-4 \mathrm{~Hz}$ oscillations and to gamma oscillations (Fig. 1E,F). Analogous trends have been observed with rodent hippocampal theta (Fox et al., 1986; Bragin et al., 1995). An additional link between these two oscillatory patterns comes from Bódizs et al. (2001), who report that during rapid-eyemovement sleep, the human hippocampus exhibits robust 1.5-3 $\mathrm{Hz}$ delta-band oscillations, whereas animals in this state display $4-8 \mathrm{~Hz}$ theta oscillations. Based on this evidence, we suggest that the frequency range of human hippocampal theta may extend to $\approx 1 \mathrm{~Hz}$.

The existence of neuronal phase locking helps to interpret behavior-related changes in brain recordings. For example, after

humans view visual stimuli, theta oscillations reset to particular phases in response to task demands (Rizzuto et al., 2003). This phase reset simultaneously occurs throughout multiple brain regions; thus, it may cause phase-locked neurons throughout the brain to spike in precise temporal patterns. Thus, our findings suggest that oscillatory phase resetting temporally synchronizes phase-locked neurons in widespread brain regions. This is consistent with reports of behavior-related changes in the coherence of oscillations in different brain regions (Jones and Wilson, 2005; Hyman et al., 2005). These patterns of inter-region oscillatory synchrony may be elaborate because our findings suggest that neuronal phase locking spans a broad range of frequencies (Fig. $2 A$ ), and that some brain regions exhibit oscillations at multiple frequencies (Fig. 1C,E,F,H) (Chrobak and Buzsáki, 1998). Finally, recent imaging studies showed that hemodynamic activity closely relates to both power of gamma oscillations and mean neuronal firing rates (Logothetis et al., 2001; Mukamel et al., 2005; Niessing et al., 2005). Our findings substantially add to these results by indicating that during high-power gamma oscillations, neuronal activity is phase locked to these oscillations. Thus, we suggest that reports of increased hemodynamic activity, in regions in which they are correlated with the amplitude of gamma oscillations, should be interpreted both in terms of increased neuronal firing and increased neuronal gamma-band synchrony.

\section{References}

Berry SD, Thompson RF (1978) Prediction of learning rate from the hippocampal electroencephalogram. Science 200:1298-1300.

Bódizs R, Kántor S, Szabó G, Szũcs A, Erõss L, Halász P (2001) Rhythmic hippocampal slow oscillation characterizes REM sleep in humans. Hippocampus $747-753$.

Bragin A, Jando G, Nadasdy Z, Hetke J, Wise K, Buzsaki G (1995) Gamma $(40-100 \mathrm{~Hz})$ oscillation in the hippocampus of the behaving rat. J Neurosci 15:47-60.

Buzsáki G (2002) Theta oscillations in the hippocampus. Neuron 33:325-340.

Buzsáki G (2005) Theta rhythm of navigation: link between path integration and landmark navigation, episodic and semantic memory. Hippocampus $15: 827-840$.

Chrobak J, Buzsáki G (1998) Gamma oscillations in the entorhinal cortex of the freely behaving rat. J Neurosci 18:388-298.

Csicsvari J, Jameison B, Wise KD, Buzsáki G (2003) Mechanisms of gamma oscillations in the hippocampus of the behaving rat. Neuron 37:311-322.

Eeckman F, Freeman W (1990) Correlations between unit firing and EEG in the rat olfactory system. Brain Res 528:238-244.

Ekstrom AD, Caplan J, Ho E, Shattuck K, Fried I, Kahana M (2005) Human hippocampal theta activity during virtual navigation. Hippocampus 15:881-889.

Fisher NI (1993) Statistical analysis of circular data. Cambridge, UK: Cambridge UP.

Fox SE, Wolfson S, Ranck JBJ (1986) Hippocampal theta rhythm and the firing of neurons in walking and urethane anesthetized rats. Exp Brain Res 62:495-508.

Freeman W, Schneider W (1982) Changes in spatial patterns of rabbit olfactory EEG with conditioning to odors. Psychophysiology 19:44-56.

Fried I, Wilson CL, Maidment NT, Engel Jr J, Behnke E, Fields TA, MacDonald KA, Morrow JW, Ackerson L (1999) Cerebral microdialysis combined with single-neuron and electroencephalographic recording in neurosurgical patients. J Neurosurg 91:697-705.

Fries P (2005) A mechanism for cognitive dynamics: neuronal communication through neuronal coherence. Trends Cogn Sci 9:474-480.

Fries P, Reynolds JH, Rorie AE, Desimone R (2001) Modulation of oscillatory neuronal synchronization by selective visual attention. Science 291:1560-1563.

Gray C, König P, Engel A, Singer W (1989) Oscillatory responses in cat visual cortex exhibit inter-columnar synchronization which reflects global stimulus properties. Nature 338:334-337. 
Harris KD, Csicsvari J, Hirase H, Dragoi G, Buzsáki G (2003) Organization of cell assemblies in the hippocampus. Nature 424:552-556.

Hasselmo ME, Bodelon C, Wyble BP (2002) A proposed function for hippocampal theta rhythm: separate phases of encoding and retrieval enhance reversal of prior learning. Neural Comput 14:793-817.

Huxter J, Burgess N, O'Keefe J (2003) Independent rate and temporal coding in hippocampal pyramidal cells. Nature 425:828-832.

Hyman J, Zilli E, Paley A, Hasselmo M (2005) Medial prefrontal cortex cells show dynamic modulation with the hippocampal theta rhythm dependent on behavior. Hippocampus 15:739-749.

Jacobs J, Hwang G, Curran T, Kahana MJ (2006) EEG oscillations and recognition memory: theta correlates of memory retrieval and decision making. NeuroImage 15:978-987.

Jones MW, Wilson MA (2005, Dec) Theta rhythms coordinate hippocampalprefrontal interactions in a spatial memory task. PLoS Biol 3:e402.

Kahana MJ (2006) The cognitive correlates of human brain oscillations. J Neurosci 26:1669-1672.

Lee H, Simpson G, Logothetis N, Rainer G (2005) Phase locking of single neuron activity to theta oscillations during working memory in monkey extrastriate visual cortex. Neuron 45:147-156.

Lisman J, Idiart MA (1995) Storage of $7 \pm 2$ short-term memories in oscillatory subcycles. Science 267:1512-1515.

Logothetis N, Pauls J, Augath M, Trinath T, Oeltermann A (2001) Neurophysio-logical investigation of the basis of the fMRI signal. Nature 412:150-157.

Mormann F, Fell J, Axmacher N, Weber B, Lehnertz K, Elger C, Fernandez G (2005) Phase/amplitude reset and theta-gamma interaction in the human medial temporal lobe during a continuous word recognition memory task. Hippocampus 15:890-900.

Mukamel R, Gelbard H, Arieli A, Hasson U, Fried I, Malach R (2005) Coupling between neuronal firing, field potentials, and FMRI in human auditory cortex. Science 309:951-954.
Niessing J, Ebisch B, Schmidt KE, Niessing M, Singer W, Galuske RA (2005) Hemodynamic signals correlate tightly with synchronized gamma oscillations. Science 309:948-951.

O'Keefe J, Recce ML (1993) Phase relationship between hippocampal place units and the EEG theta rhythm. Hippocampus 3:317-330.

Pesaran B, Pezarais JS, Sahani M, Mitra PP, Andersen RA (2002) Temporal structure in neuronal activity during working memory in macaque parietal cortex. Nat Neurosci 5:805-811.

Pike F, Goddard RS, Suckling JM, Ganter P, Kasthuri N, Paulsen O (2000) Distinct frequency preferences of different types of rat hippocampal neurones in response to oscillatory input currents. J Physiol (Lond) 529:205-213.

Quiroga RQ, Nadasdy Z, Ben-Shaul Y (2004) Unsupervised spike detection and sorting with wavelets and superparamagnetic clustering. Neural Comput 16:1661-1687.

Raghavachari S, Lisman JE, Tully M, Madsen JR, Bromfield EB, Kahana MJ (2006) Theta oscillations in human cortex during a working memory task: evidence for local generators. J Neurophysiol 95:1630-1638.

Rizzuto DS, Madsen JR, Bromfield EB, Schulze-Bonhage A, Seelig D, Aschenbrenner-Scheibe R, Kahana MJ (2003) Reset of human neocortical oscillations during a working memory task. Proc Natl Acad Sci USA 100:7931-7936

Siapas A, Lubenov E, Wilson M (2005) Prefrontal phase locking to hippocampal theta oscillations. Neuron 46:141-151.

Skaggs WE, McNaughton BL, Wilson MA, Barnes CA (1996) Theta phase precession in hippocampal neuronal populations and the compression of temporal sequences. Hippocampus 6:149-172.

Witter M, Wouterlood F (2002) The parahippocampal region: past, present and future. In: The parahippocampal region: organization and role in cognitive functions (Witter M, Wouterlood F, eds), pp 3-19. Oxford: Oxford UP. 